\title{
COMPOSITION OPERATORS BETWEEN ANALYTIC CAMPANATO SPACES
}

\author{
JIE XIAO AND WEN XU
}

\begin{abstract}
Aвstract. This note characterizes both boundedness and compactness of a composition operator between any two analytic Campanato spaces on the unit complex disk.
\end{abstract}

\section{INTRODUCTION}

On the basis of the works: [15], [5], [6, 7], [8], [18], [19], [17], [1], [9, 10], [12], [3], [20, 21, 22], [13, 14] and [2], we consider an unsolved fundamental problem in the function-theoretic operator theory, i.e., the socalled composition operator question for the analytic Campanato spaces:

Question 1. Let $\phi$ be an analytic self-map of $\mathbb{D}$ and $-\infty<p, q<\infty$. What finite (resp. vanishing) property must $\phi$ have in order that $C_{\phi}$ is bounded (resp. compact) between $\subset \mathcal{A}_{p}$ and $\subset \mathcal{A}_{q}$ ?

In the above and below, $\mathbb{D}$ and $\mathbb{T}$ respectively represent the unit disk and the unit circle in the finite complex plane $\mathbb{C}, C_{\phi} f=f \circ \phi$ is the composition of an analytic function $f$ on $\mathbb{D}$ with $\phi$, and for $p \in(-\infty, \infty)$, and $C \mathcal{A}_{p}$ denotes the so-called Campanato space of all analytic functions $f: \mathbb{D} \rightarrow \mathbb{C}$ with radial boundary values $f$ on $\mathbb{T}$ satisfying

$$
\|f\|_{C \mathcal{A}_{p}}=\sup _{I \subseteq \mathbb{T}} \sqrt{|I|^{-p} \int_{I}\left|f(\xi)-f_{I}\right|^{2}|d \xi|}<\infty
$$

where the supremum is taken over all sub-arcs $I \subseteq \mathbb{T}$ with $|I|$ being their arc-lengths, and

$$
|d \zeta|=\left|d e^{i \theta}\right|=d \theta ; \quad f_{I}=|I|^{-1} \int_{I} f(\xi)|d \xi| .
$$

Neededless to say, $\|\cdot\|_{\mathcal{C} \mathcal{A}_{p}}$ cannot distinguish between any two $C \mathcal{A}_{p}$ functions differing by a constant, but $|f(0)|+\|\cdot\|_{\mathcal{C} \mathcal{A}_{p}}$ defines a norm so that $C \mathcal{A}_{p}$

2000 Mathematics Subject Classification. 30H10, 30H25, 30H35, 46E15, 47B33.

Key words and phrases. Composition operator, boundedness, compactness, analytic Campanato space.

JX and WX were in part supported by NSERC of Canada and the Finnish Cultural Foundation, respectively. 
is a Banach space. Here, it is perhaps appropriate to mention the following table which helps us get a better understanding of the structure of $C \mathcal{A}_{p}$ (see, e.g. [4, pp. 67-75] and [22, p. 52]):

\begin{tabular}{|l|l|}
\hline Index $p$ & Analytic Campanato Space $\mathcal{C A}_{p}$ \\
\hline$p \in(-\infty, 0]$ & Analytic Hardy space $\mathcal{H}^{2}$ \\
\hline$p \in(0,1)$ & Holomorphic Morrey space $\mathcal{H}^{2, p}$ \\
\hline$p=1$ & Analytic John-Nirenberg space $\mathcal{B} \mathcal{M O} \mathcal{A}$ \\
\hline$p \in(1,3]$ & Analytic Lipschitz space $\mathcal{A}_{\frac{p-1}{2}}$ \\
\hline$p \in(3, \infty)$ & Complex constant space $\mathbb{C}$ \\
\hline
\end{tabular}

An answer to the boundedness part of Question 1 is the following result.

Theorem 1. Let $\phi$ be an analytic self-map of $\mathbb{D}$ and $(p, q) \in[0,2) \times[0,2)$. Then $C_{\phi}: C \mathcal{A}_{p} \mapsto C \mathcal{A}_{q}$ is bounded if and only if

$$
\sup _{a \in \mathbb{D}} \frac{\left(1-|a|^{2}\right)^{1-q}}{\left(1-|\phi(a)|^{2}\right)^{1-p}}\left\|\sigma_{\phi(a)} \circ \phi \circ \sigma_{a}\right\|_{2}^{2}<\infty
$$

where

$$
\sigma_{b}(z)=\frac{b-z}{1-\bar{b} z} \quad \& \quad\|f\|_{2}=\sqrt{\int_{\mathbb{T}}|f(\xi)|^{2}|d \xi|} .
$$

It should be pointed out that (1) is not always true - in fact, we have the following consequence whose (i) with $p=q \in\{0,1\}$ and (ii) are wellknown; see e.g. [9, 12, 13, 14, 15, 20].

Corollary 1. Let $\phi$ be an analytic self-map of $\mathbb{D}$. For $f \in \mathcal{H}^{2}$ and $p \in[0,2)$ set

$$
\|f\|_{C \mathcal{A}_{p}, *}=\sup _{a \in \mathbb{D}}\left(1-|a|^{2}\right)^{\frac{1-p}{2}}\left\|f \circ \sigma_{a}-f(a)\right\|_{2} .
$$

(i) If $p \in[0,1]$ then $C_{\phi}: C \mathcal{A}_{p} \mapsto C \mathcal{A}_{p}$ is always bounded with

$$
\left\|C_{\phi} f\right\|_{C \mathcal{A}_{p}, *} \leq\left(\frac{1+|\phi(0)|}{1-|\phi(0)|}\right)^{\frac{1-p}{2}}\|f\|_{C \mathcal{A}_{p}, *} .
$$

(ii) If $p \in(1,2)$ then $C_{\phi}: C \mathcal{A}_{p} \mapsto C \mathcal{A}_{p}$ is bounded when and only when

$$
\sup _{a \in \mathbb{D}}\left(\frac{1-|a|^{2}}{1-|\phi(a)|^{2}}\right)^{\frac{3-p}{2}}\left|\phi^{\prime}(a)\right|<\infty .
$$

Below is a partial answer to the compactness part of Question 1 ,

Theorem 2. Let $\phi$ be an analytic self-map of $\mathbb{D}$ and $(p, q) \in[0,2) \times[0,2)$. If $C_{\phi}: C \mathcal{A}_{p} \mapsto C \mathcal{A}_{q}$ is compact then (1) holds and

$$
\lim _{|\phi(a)| \rightarrow 1} \frac{\left(1-|a|^{2}\right)^{1-q}}{\left(1-|\phi(a)|^{2}\right)^{1-p}}\left\|\sigma_{\phi(a)} \circ \phi \circ \sigma_{a}\right\|_{2}^{2}=0 .
$$


Conversely, if (1) holds and (4) is valid for $(p, q) \in[0,2) \times[1,1] \cup(1,2) \times$ $[0,2)$ then $C_{\phi}: C \mathcal{A}_{p} \mapsto C \mathcal{A}_{q}$ is compact.

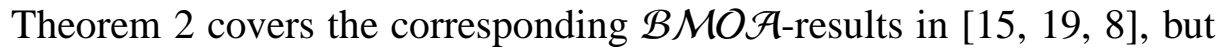
also it derives the following assertion extending the known one in [12, 10 , 20].

Corollary 2. Let $\phi$ be an analytic self-map of $\mathbb{D}$ and $p \in[0,2)$. If $C_{\phi}$ : $C \mathcal{A}_{p} \mapsto C \mathcal{A}_{p}$ is compact then (3) holds and

$$
\lim _{|\phi(a)| \rightarrow 1}\left(\frac{1-|a|^{2}}{1-|\phi(a)|^{2}}\right)^{\frac{3-p}{2}}\left|\phi^{\prime}(a)\right|=0 .
$$

Conversely, if (3) holds and (5) is valid for $p \in(1,2)$ then $C_{\phi}: C \mathcal{A}_{p} \mapsto C \mathcal{A}_{p}$ is compact.

Conjecture 1. The converse part of Theorem 2 still holds for $(p, q) \in$ $[0,2) \times[0,2) \backslash([0,2) \times[1,1] \cup(1,2) \times[0,2))$.

Notation: From now on, $\mathrm{X} \lesssim \mathrm{Y}, \mathrm{X} \gtrsim \mathrm{Y}$, and $\mathrm{X} \approx \mathrm{Y}$ represent that there exists a constant $\kappa>0$ such that $\mathrm{X} \leq \kappa \mathrm{Y}, \mathrm{X} \geq \kappa \mathrm{Y}$, and $\kappa^{-1} \mathrm{Y} \leq \mathrm{X} \leq \kappa \mathrm{Y}$, respectively. In addition, $d m$ stands for two dimensional Lebesgue measure.

\section{BoundEDNESS}

In order to prove Theorem 1 and Corollary 1, we need two lemmas.

Lemma 1. Let $p \in[0,2)$ and $f \in \mathcal{H}^{2}$. Then $f \in C \mathcal{A}_{p}$ if and only if $\|f\|_{C \mathcal{A}_{p}, *}<\infty$.

Proof. Case 1: $p=0$. This is trivial.

Case 2: $p \in(0,1]$. This situation can be verified by [22, Theorem 3.2.1] and the well-known Hardy-Littlewood identity for $f \in \mathcal{H}^{2}$ :

$$
\pi^{-1} \int_{\mathbb{D}}\left|f^{\prime}(z)\right|^{2}\left(-\ln |z|^{2}\right) d m(z)=(2 \pi)^{-1} \int_{\mathbb{T}}|f(\xi)-f(0)|^{2}|d \xi| .
$$

Case 3: $p \in(1,2)$. Let $g=f \circ \sigma_{a}-f(a)$. Then

(7) $\quad\left(1-|a|^{2}\right)\left|f^{\prime}(a)\right|=\left|g^{\prime}(0)\right| \leq(2 \pi)^{-1 / 2}\|g\|_{2}=(2 \pi)^{-1 / 2}\left\|f \circ \sigma_{a}-f(a)\right\|_{2}$.

If $f \in C \mathcal{A}_{p}$ then an application of (7) yields

$$
\sup _{a \in \mathbb{D}}\left(1-|a|^{2}\right)^{\frac{3-p}{2}}\left|f^{\prime}(a)\right|<\infty
$$

and consequently, $f \in \mathcal{A}_{\frac{p-1}{2}}$, as desired. Conversely, if $f \in \mathcal{A}_{\frac{p-1}{2}}$ then

$$
\mathrm{A}=\sup _{\xi_{1} \neq \xi_{2} \text { in } \mathbb{D U T}} \frac{\left|f\left(\xi_{1}\right)-f\left(\xi_{2}\right)\right|}{\left|\xi_{1}-\xi_{2}\right|^{\frac{p-1}{2}}}<\infty .
$$


This, along with $p \in(1,2)$ and [23, p. 63, Ex. 8], gives

$$
\begin{aligned}
& \|f\|_{C \mathcal{A}_{p}, *}^{2} \\
& =\sup _{a \in \mathbb{D}}\left(1-|a|^{2}\right)^{1-p} \int_{\mathbb{T}}\left|f \circ \sigma_{a}(\xi)-f(a)\right|^{2}|d \xi| \\
& \lesssim \mathrm{A}^{2} \sup _{a \in \mathbb{D}} \int_{\mathbb{T}}\left(\frac{1-|a|^{2}}{\left|\sigma_{a}(\xi)-a\right|}\right)^{1-p}|d \xi| \\
& \approx \mathrm{A}^{2} \sup _{a \in \mathbb{D}} \int_{\mathbb{T}} \frac{\left(1-|a|^{2}\right)^{2-p}}{|1-\bar{a} \eta|^{3-p}}|d \eta| \\
& \lesssim \mathrm{A}^{2} .
\end{aligned}
$$

Lemma 2. For $p \in[0,2)$ let $f_{b}(z)=\left(1-|b|^{2}\right)^{\frac{1+p}{2}} /(1-\bar{b} z)$. Then $f_{b}$ is uniformly bounded in $C \mathcal{A}_{p}$, i.e., $\sup _{b \in \mathbb{D}}\left\|f_{b}\right\|_{C \mathcal{A}_{p, *}}<\infty$.

Proof. Using [11, Lemma 2.5], we get the following estimate:

$$
\begin{aligned}
& \mathrm{B} \\
& =\int_{\mathbb{D}}\left|f_{b}^{\prime}(z)\right|^{2}\left(1-\left|\sigma_{a}(z)\right|^{2}\right) d m(z) \\
& =\left(|b|\left(1-|b|^{2}\right)^{\frac{1+p}{2}}\right)^{2}\left(1-|a|^{2}\right) \int_{\mathbb{D}} \frac{1-|z|^{2}}{|1-\bar{a} z|^{2}|1-\bar{b} z|^{4}} d m(z) \\
& \lesssim \frac{\left(|b|\left(1-|b|^{2}\right)^{\frac{1+p}{2}}\right)^{2}\left(1-|a|^{2}\right)}{\left(1-|b|^{2}\right)|1-\bar{a} b|^{2}} .
\end{aligned}
$$

Choosing $a=\sigma_{b}(c)$, we utilize $1-|z| \lesssim-\ln |z|$ to obtain that if $p \in[0,2)$ then

$$
\begin{aligned}
& \left\|f_{b}\right\|_{\mathcal{C A}_{p}, *}^{2} \\
& \lesssim \sup _{a \in \mathbb{D}}\left(1-|a|^{2}\right)^{1-p} \mathrm{~B} \\
& \lesssim \sup _{a \in \mathbb{D}} \frac{\left(1-|a|^{2}\right)^{2-p}\left(1-|b|^{2}\right)^{p}}{|1-\bar{a} b|^{2}} \\
& =\sup _{c \in \mathbb{D}}\left(\frac{1-|c|^{2}}{|1-\bar{b} c|}\right)^{2-p}|1-\bar{b} c|^{p} \\
& \leq 2^{2}
\end{aligned}
$$

as desired.

Proof of Theorem 1]. Using [6, Proposition 2.3], we have that if $g(0)=0=$ $\psi(0), g \in \mathcal{H}^{2}$, and $\psi$ is an analytic self-map of $\mathbb{D}$, then

$\|g \circ \psi\|_{2} \lesssim\|g\|_{2}\|\psi\|_{2}$. 
Setting

$$
g_{a}=f \circ \sigma_{\phi(a)}-f \circ \phi(a) \quad \& \quad \psi_{a}=\sigma_{\phi(a)} \circ \phi \circ \sigma_{a},
$$

we get

$$
g_{a} \circ \psi_{a}=f \circ \phi \circ \sigma_{a}-f \circ \phi(a)
$$

As a consequence of Lemma 1 and (9), we find that if (1) is valid then

$$
\begin{aligned}
& \left\|C_{\phi} f\right\|_{C \mathcal{A}_{q}, *}^{2} \\
& =\sup _{a \in \mathbb{D}}\left(1-|a|^{2}\right)^{1-q}\left\|f \circ \phi \circ \sigma_{a}-f \circ \phi(a)\right\|_{2}^{2} \\
& \lesssim \sup _{a \in \mathbb{D}} \frac{\left(1-|a|^{2}\right)^{1-q}}{\left(1-|\phi(a)|^{2}\right)^{1-p}}\left(\left(1-|\phi(a)|^{2}\right)^{1-p}\left\|g_{a}\right\|_{2}^{2}\right)\left\|\psi_{a}\right\|_{2}^{2} \\
& \lesssim\|f\|_{C \mathcal{A}_{p}, *}^{2} \sup _{a \in \mathbb{D}} \frac{\left(1-|a|^{2}\right)^{1-q}}{\left(1-|\phi(a)|^{2}\right)^{1-p}}\left\|\psi_{a}\right\|_{2}^{2},
\end{aligned}
$$

and consequently, $C_{\phi}$ exists as a bounded operator from $C \mathcal{A}_{p}$ into $C \mathcal{A}_{q}$.

For the "only-if" part, recall the so-called Navanlinna counting function of $\phi$ :

$$
N(\phi, w)=\sum_{z: \phi(z)=w} \ln |z|^{-1} \quad \forall \quad w \in \mathbb{D} \backslash\{\phi(0)\}
$$

and the associated change of variable formula:

$$
\int_{\mathbb{D}}\left|\left(C_{\phi} f\right)^{\prime}(z)\right|^{2} \ln |z|^{-1} d m(z)=\int_{\mathbb{D}}\left|f^{\prime}(w)\right|^{2} N(\phi, w) d m(w) \quad \forall f \in \mathcal{H}^{2}
$$

A combination of (10) and (6) gives that if $b=\phi(a)$ then

$$
\left\|\sigma_{b} \circ \phi \circ \sigma_{a}\right\|_{2}^{2}=4 \int_{\mathbb{D}} N\left(\sigma_{b} \circ \phi \circ \sigma_{a}, z\right) d m(z)
$$

Now, if $C_{\phi}: C \mathcal{A}_{p} \mapsto C \mathcal{A}_{q}$ is bounded, then the test function $f_{b}$ in Lemma 2 is used to imply

$$
\mathrm{C}=\sup _{a, b \in \mathbb{D}}\left(1-|a|^{2}\right)^{1-q} \int_{\mathbb{D}}\left|\left(f_{b} \circ \phi \circ \sigma_{a}\right)^{\prime}(z)\right|^{2} \ln |z|^{-1} d m(z)<\infty .
$$


and consequently,

$$
\begin{aligned}
& \text { C } \\
& \gtrsim \sup _{a, b \in \mathbb{D}}\left(1-|a|^{2}\right)^{1-q}|b|^{2}\left(1-|b|^{2}\right)^{p-1} \int_{\mathbb{D}}\left|\sigma_{b}^{\prime}(z)\right|^{2} N\left(\phi \circ \sigma_{a}, z\right) d m(z) \\
& \gtrsim \sup _{a \in \mathbb{D}} \frac{\left(1-|a|^{2}\right)^{1-q}|\phi(a)|^{2}}{\left(1-|\phi(a)|^{2}\right)^{1-p}} \int_{\mathbb{D}} N\left(\sigma_{\phi(a)} \circ \phi \circ \sigma_{a}, z\right) d m(z) \\
& \gtrsim \sup _{a \in \mathbb{D}} \frac{\left(1-|a|^{2}\right)^{1-q}|\phi(a)|^{2}}{\left(1-|\phi(a)|^{2}\right)^{1-p}}\left\|\sigma_{\phi(a)} \circ \phi \circ \sigma_{a}\right\|_{2}^{2} \\
& \gtrsim s^{2} \sup _{a \in \mathbb{D}} \frac{\left(1-|a|^{2}\right)^{1-q}}{\left(1-|\phi(a)|^{2}\right)^{1-p}}\left\|\sigma_{\phi(a)} \circ \phi \circ \sigma_{a}\right\|_{2}^{2}
\end{aligned}
$$

as $|\phi(a)|>s \in(0,1)$. Note also that the identity map $f(z)=z$ is an element of $C \mathcal{A}_{p}$. Thus, boundedness of $C_{\phi}: C \mathcal{A}_{p} \mapsto C \mathcal{A}_{q}$ ensures $\|\phi\|_{C \mathcal{A}_{q, *}}<\infty$, and consequently, if $|\phi(a)| \leq s<1$ then

$$
\begin{aligned}
& \sup _{a \in \mathbb{D}} \frac{\left(1-|a|^{2}\right)^{1-q}}{\left(1-|\phi(a)|^{2}\right)^{1-p}}\left\|\sigma_{\phi(a)} \circ \phi \circ \sigma_{a}\right\|_{2}^{2} \\
& \lesssim\left(1+(1-s)^{p-1}\right) \sup _{a \in \mathbb{D}}\left(1-|a|^{2}\right)^{1-q} \int_{\mathbb{T}}\left|\frac{\phi(a)-\phi \circ \sigma_{a}(\xi)}{1-\overline{\phi(a)} \phi \circ \sigma_{a}(\xi)}\right|^{2}|d \xi| \\
& \lesssim\left(\frac{1+(1-s)^{p-1}}{(1-s)^{2}}\right) \sup _{a \in \mathbb{D}}\left(1-|a|^{2}\right)^{1-q}\left\|\phi(a)-\phi \circ \sigma_{a}\right\|_{2}^{2} \\
& \approx\left(\frac{1+(1-s)^{p-1}}{(1-s)^{2}}\right)\|\phi\|_{C \mathcal{A}_{q}, *}^{2} .
\end{aligned}
$$

The above estimates imply (1).

Proof of Corollary 1$]$ (i) Under $p \in[0,1]$, we use the Schwarz lemma for $\sigma_{\phi(0)} \circ \phi$ to deduce that (1) holds for $p=q \in[0,1]$, and so that $C_{\phi}$ is bounded on $C \mathcal{A}_{p}$ due to Theorem 1. To reach (2), let us begin with the case $\phi(0)=0$. According to the setting in the argument for Theorem 1, the well-known Littlewood subordination principle and Schwarz's lemma for $\phi$, we have

$$
\begin{aligned}
& \left\|C_{\phi} f\right\|_{C \mathcal{A}_{p}, *}^{2} \\
& =\sup _{a \in \mathbb{D}}\left(1-|a|^{2}\right)^{1-p}\left\|g_{a} \circ \psi_{a}\right\|_{2}^{2} \\
& \leq \sup _{a \in \mathbb{D}}\left(1-|a|^{2}\right)^{1-p}\left\|g_{a}\right\|_{2}^{2} \\
& \leq\|f\|_{\mathcal{C A}_{p}, *}^{2} \sup _{a \in \mathbb{D}}\left(\frac{1-|a|^{2}}{1-|\phi(a)|^{2}}\right)^{1-p} \\
& \leq\|f\|_{\mathcal{C A}_{p}, *}^{2}
\end{aligned}
$$


Next, for the general case let

$$
\left\{\begin{array}{l}
\psi=\sigma_{\phi(0)} \circ \phi ; \\
\lambda=\frac{\bar{a} b-1}{1-a \bar{b}} ; \\
b=\phi(0) ; \\
c=\sigma_{a}(b) .
\end{array}\right.
$$

Then $\psi(0)=0$ and thus

$$
\begin{aligned}
& \left\|C_{\sigma_{b}} f\right\|_{\mathcal{C} \mathcal{A}_{p}, *}^{2} \\
& =\sup _{a \in \mathbb{D}}\left(1-|a|^{2}\right)^{1-p}\left\|f\left(\lambda \sigma_{c}\right)-f(\lambda c)\right\|_{2}^{2} \\
& \leq\|f\|_{C \mathcal{A}_{p}, *}^{2} \sup _{a \in \mathbb{D}}\left(\frac{1-|a|^{2}}{1-|c|^{2}}\right)^{1-p} \\
& \leq\|f\|_{C \mathcal{A}_{p}, *}^{2}\left(\frac{1+|b|}{1-|b|}\right)^{1-p} .
\end{aligned}
$$

Using the previous estimates, we get

$$
\left\|C_{\phi} f\right\|_{\mathcal{C A}_{p}, *}^{2}=\left\|f \circ \sigma_{b} \circ \psi\right\|_{\mathcal{C A}_{p}, *}^{2} \leq\left\|f \circ \sigma_{b}\right\|_{\mathcal{C A}_{p}, *}^{2} \leq\|f\|_{\mathcal{C A}_{p}, *}^{2}\left(\frac{1+|b|}{1-|b|}\right)^{1-p},
$$

whence reaching (2).

(ii) Suppose $p \in(1,2)$. Then (7) yields

$$
\sup _{a \in \mathbb{D}}\left(\frac{1-|a|^{2}}{1-|\phi(a)|^{2}}\right)^{3-p}\left|\phi^{\prime}(a)\right|^{2} \leq \sup _{a \in \mathbb{D}}\left(\frac{1-|a|^{2}}{1-|\phi(a)|^{2}}\right)^{1-p}\left\|\sigma_{\phi(a)} \circ \phi \circ \sigma_{a}\right\|_{2}^{2},
$$

so, if $C_{\phi}$ is bounded on $C \mathcal{A}_{p}$ then (1) holds with $p=q$ due to Theorem 1 , and hence (3) holds. Conversely, if (3) is true, then $C_{\phi}$ is bounded on $\mathcal{A}_{\frac{p-1}{2}}$ (cf. [9, Theorem A]) and hence bounded on $C \mathcal{A}_{p}$.

\section{Compactness}

The arguments for Theorem 2 and Corollary 2 depend on the two basic facts below.

Lemma 3. Let $p \in(0,2)$ and $f \in C \mathcal{A}_{p}$ with $f(0)=0$. Then

$$
\int_{\mathbb{T}}|f(\xi)|^{4}|d \xi| \lesssim\left\{\begin{array}{l}
\|f\|_{C \mathcal{A}_{p}, *}^{2}\|f\|_{2}^{2} \text { for } p \in[1,2) ; \\
\|f\|_{C \mathcal{A}_{p}, *}^{2} \int_{0}^{\infty} t H_{\infty}^{p}(\{\xi \in \mathbb{T}:|f(\xi)|>t\}) d t \text { for } p \in(0,1],
\end{array}\right.
$$

where $H_{\infty}^{p}(E)=\inf _{E \subseteq \cup_{I_{j}}} \sum_{j}\left|I_{j}\right|^{p}$ is p-dimensional Hausdorff capacity of $E \subseteq$ $\mathbb{T}$ - the infimum is taken over all arc coverings $\cup_{j} I_{j} \supseteq E$. 
Proof. Let $d \mu=\left|f^{\prime}(z)\right|^{2}\left(1-|z|^{2}\right) d m(z)$. From $f \in C \mathcal{A}_{p}$ it follows that $\mu$ is a $p$-Carleson measure on $\mathbb{D}$ - in other words -

$$
\|\mu\|_{\mathcal{C M}_{p}}=\sup _{I \subseteq \mathbb{T}}|I|^{-p} \mu(S(I)) \lesssim\|f\|_{C \mathcal{A}_{p}, *}^{2}
$$

where $S(I)=\left\{z=r e^{i \theta} \in \mathbb{D}: 1-|I| /(2 \pi) \leq r<1 \&\left|\theta-\theta_{I}\right| \leq|I| / 2\right\}$ is the Carleson box based on the arc $I \subseteq \mathbb{T}$ taking $\theta_{I}$ as its center. In fact, if $a=(1-|I| /(2 \pi)) e^{i\left(\theta_{I}+|I| / 4\right)}$ then a simple computation, along with (6) and $-\ln |z| \approx 1-|z|^{2}$ as $|z| \geq 2^{-1}$ as well as Lemma 1, gives

$$
\begin{aligned}
& |I|^{-p} \mu(S(I)) \\
& \lesssim\left(1-|a|^{2}\right)^{1-p} \int_{S(I)}\left|f^{\prime}(z)\right|^{2}\left(1-\left|\sigma_{a}(z)\right|^{2}\right) d m(z) \\
& \lesssim\left(1-|a|^{2}\right)^{1-p}\left\|f \circ \sigma_{a}-f(a)\right\|_{2}^{2} \\
& \lesssim\|f\|_{C \mathcal{A}_{p}, *}^{2}
\end{aligned}
$$

In particular, when $p \in(1,2), \mu$ is also 1-Carleson measure with $\|\mu\|_{\mathcal{C M}_{1}} \lesssim$ $\|\mu\|_{\mathcal{C M}_{p}}$. According to [22, p. 79, Theorem 4.1.4], we have

$$
\int_{\mathbb{D}}|f|^{2} d \mu \lesssim\|\mu\|_{\mathcal{C M}_{p}}\left\{\begin{array}{l}
\|f\|_{2}^{2} \text { for } p \in[1,2) \\
\int_{0}^{\infty} t H_{\infty}^{p}(\{\xi \in \mathbb{T}:|f(\xi)|>t\}) d t \text { for } p \in(0,1] .
\end{array}\right.
$$

This last estimate, along with the following Hardy-Stein identity based estimate (cf. [22, p. 36])

$$
\begin{aligned}
& \int_{\mathbb{T}}|f(\xi)|^{4}|d \xi| \\
& \approx \int_{\mathbb{D}}|f(z)|^{2}\left|f^{\prime}(z)\right|^{2}\left(\ln |z|^{-1}\right) d m(z) \\
& \lesssim \int_{\mathbb{D}}|f(z)|^{2}\left|f^{\prime}(z)\right|^{2}\left(1-|z|^{2}\right) d m(z) \\
& \approx \int_{\mathbb{D}}|f|^{2} d \mu,
\end{aligned}
$$

implies the desired estimate.

Lemma 4. Let $(p, q) \in[0,2) \times[1,1]$. If an analytic self-map $\phi$ of $\mathbb{D}$ satisfies (4), then one has

$$
\lim _{t \rightarrow 1} \sup _{|\phi(a)| \leq s} \frac{\left(1-|a|^{2}\right)^{1-q}}{\left(1-|\phi(a)|^{2}\right)^{1-p}}\left|\left\{\xi \in \mathbb{T}:\left|\sigma_{\phi(a)} \circ \phi \circ \sigma_{a}(\xi)\right|>t\right\}\right|=0 \forall s \in(0,1) .
$$

Proof. Note that

$$
\left|\phi \circ \sigma_{a}\right| \rightarrow 1 \Longleftrightarrow\left|\sigma_{\phi(a)} \circ \phi \circ \sigma_{a}\right| \rightarrow 1 \quad \text { under } \quad|\phi(a)| \leq s .
$$


So, it suffices to show that (4) implies

$$
\lim _{t \rightarrow 1} \sup _{|\phi(a)| \leq s} \frac{\left(1-|a|^{2}\right)^{1-q}}{\left(1-|\phi(a)|^{2}\right)^{1-p}}\left|\left\{\xi \in \mathbb{T}:\left|\phi \circ \sigma_{a}(\xi)\right|>t\right\}\right|=0 \forall s \in(0,1) .
$$

Following [8], for $r e^{i \theta} \in \mathbb{D}$ let

$$
J\left(r e^{i \theta}\right)=\left\{e^{i t}:|t-\theta| \leq \pi(1-r)\right\} .
$$

Clearly, $J\left(r e^{i \theta}\right)$ is the sub-arc of $\mathbb{T}$ centered at $e^{i \theta}$. Importantly, [8, Lemma $3]$ tells us that for any measurable set $E \subseteq \mathbb{T}$ with 1-dimensional Lebesgue measure $|E|>0$ there exists a measurable set $F \subseteq E$ such that $|F|>0$ and

$$
\frac{|J(r \xi) \cap E|}{|J(r \xi)|} \geq\left(2^{4} \pi\right)^{-1}|E| \quad \forall \quad r \in[0,1) \& \xi \in F .
$$

Suppose now (4) is valid but (12) is not true. On the one hand, we have that for any $\epsilon>0$ there is an $s \in(0,1)$ such that

$$
\frac{\left(\frac{2 \pi}{|J(a)|}\right) \int_{J(a)} \rho\left(\phi \circ \sigma_{b}(\xi), \phi \circ \sigma_{b}(a)\right)^{2}|d \xi|}{\left(1-\left|\phi \circ \sigma_{b}(a)\right|^{2}\right)^{1-p}\left(1-\left|\sigma_{b}(a)\right|^{2}\right)^{q-1}}<\epsilon \quad \forall\left|\phi \circ \sigma_{b}(a)\right|>s .
$$

Here we have used the pseudo-hyperbolic distance $\rho(z, w)=\left|\sigma_{w}(z)\right|$ between $z, w \in \mathbb{D}$ and the following basic estimate

$$
\left\{\begin{array}{l}
\left\|\sigma_{\phi(a)} \circ \phi \circ \sigma_{a}\right\|_{2}^{2}=\int_{\mathbb{T}} \rho(\phi(\xi), \phi(a))^{2} P_{a}(\xi)|d \xi| \\
P_{a}(\xi)=\left|\sigma_{a}^{\prime}(\xi)\right| \geq 2^{-1} \pi|J(a)|^{-1} \forall \xi \in J(a) .
\end{array}\right.
$$

On the other hand, we can select two constants $s_{0} \in(0,1)$ and $\epsilon_{0}>0$, points $b_{j} \in \mathbb{D}$, and numbers $t_{j} \in(0,1)$ with $\lim _{j \rightarrow \infty} t_{j}=1$ such that for any $j=1,2, \ldots$ one has $\left|\phi\left(b_{j}\right)\right| \leq s_{0}$ and

$$
E_{j}=\left\{\xi \in \mathbb{T}: \phi_{j}(\xi)=\phi \circ \sigma_{b_{j}}(\xi) \text { exists as radial limit and }\left|\phi_{j}(\xi)\right|>t_{j}\right\}
$$

obeys

$$
\left(\frac{\left(1-\left|b_{j}\right|^{2}\right)^{1-q}}{\left(1-\left|\phi\left(b_{j}\right)\right|^{2}\right)^{1-p}}\right)(2 \pi)^{-1}\left|E_{j}\right| \geq \epsilon_{0} .
$$

This (15), plus the above-stated lemma on (13), ensures that one can choose sets $F_{j} \subseteq E_{j}$ such that $\left|F_{j}\right|>0$ and

$$
\begin{aligned}
& \left(\frac{\left(1-\left|b_{j}\right|^{2}\right)^{1-q}}{\left(1-\left|\phi\left(b_{j}\right)\right|^{2}\right)^{1-p}}\right) \frac{\left|J(r \xi) \cap E_{j}\right|}{|J(r \xi)|} \\
& \geq\left(\frac{\left(1-\left|b_{j}\right|^{2}\right)^{1-q}}{\left(1-\left|\phi\left(b_{j}\right)\right|^{2}\right)^{1-p}}\right)\left(\frac{\left|E_{j}\right|}{2^{4} \pi}\right) \\
& \geq 2^{-3} \epsilon_{0} \quad \forall r \in[0,1) \& \xi \in F_{j} .
\end{aligned}
$$


If $\epsilon=2^{-4} \epsilon_{0}$ in (14), then one can take such $s$ that $s_{0}<s<1$ and (14) is true for $\left|\phi \circ \sigma_{b}(a)\right|>s$. Assuming $t_{j} \geq s$ and recalling that the definition of $E_{j}$ ensures

$$
\left|\phi \circ \sigma_{b_{j}}(r \xi)\right| \rightarrow\left|\phi \circ \sigma_{b_{j}}(\xi)\right|>t_{j} \quad \text { as } r \rightarrow 1 \text { for each } \xi \in E_{j} .
$$

Of course, this last property is valid for arbitrarily chosen point $\xi_{j} \in F_{j}$. Note that $\left|\phi \circ \sigma_{b_{j}}(0)\right|=\left|\phi\left(b_{j}\right)\right| \leq s_{0}$. Thus, by continuity of $\left|\phi \circ \sigma_{b_{j}}\right|$ there exists an $r_{j} \in(0,1)$ such that $\left|\phi \circ \sigma_{b_{j}}\left(r_{j} \xi_{j}\right)\right|=s$. If $a_{j}=r_{j} \xi_{j}$ then

$$
\rho\left(\phi \circ \sigma_{b_{j}}(\xi), \phi \circ \sigma_{b_{j}}\left(a_{j}\right)\right) \geq \rho\left(t_{j}, s\right) \quad \forall \quad \xi \in E_{j},
$$

and hence (16) and $q=1$ are applied to deduce

$$
\begin{aligned}
& \frac{\left(1-\left|\sigma_{b_{j}}\left(a_{j}\right)\right|^{2}\right)^{1-q}}{\left(1-\left|\phi \circ \sigma_{b_{j}}\left(a_{j}\right)\right|^{2}\right)^{1-p}} \int_{J\left(a_{j}\right)} \rho\left(\phi \circ \sigma_{b_{j}}(\xi), \phi \circ \sigma_{b_{j}}\left(a_{j}\right)\right)^{2} \frac{|d \xi|}{(2 \pi)^{-1}\left|J\left(a_{j}\right)\right|} \\
& \geq\left(\frac{\left(1-\left|b_{j}\right|^{2}\right)^{1-q}}{\left(1-\left|\phi\left(b_{j}\right)\right|^{2}\right)^{1-p}}\right)\left(\frac{\left|J\left(a_{j}\right) \cap E_{j}\right|}{\left|J\left(a_{j}\right)\right|}\right)\left(\frac{1-\left|\phi\left(b_{j}\right)\right|^{2}}{1-\left|\phi \circ \sigma_{b_{j}}\left(a_{j}\right)\right|^{2}}\right)^{1-p} \rho\left(t_{j}, s\right)^{2} \\
& \geq 2^{-3} \epsilon_{0}\left(\frac{\min \left\{1,\left(1-s_{0}^{2}\right)^{1-p}\right\}}{\left(1-s^{2}\right)^{1-p}}\right) \rho\left(t_{j}, s\right)^{2} .
\end{aligned}
$$

Since $\lim _{j \rightarrow \infty} \rho\left(t_{j}, s\right)=1$, it follows from (14) that

0

$$
\begin{aligned}
& =\lim _{j \rightarrow \infty} \frac{\left(1-\left|\sigma_{b_{j}}\left(a_{j}\right)\right|^{2}\right)^{1-q}}{\left(1-\left|\phi \circ \sigma_{b_{j}}\left(a_{j}\right)\right|^{2}\right)^{1-p}} \int_{J\left(a_{j}\right)}\left(\frac{\rho\left(\phi \circ \sigma_{a_{j}}(\xi), \phi \circ \sigma_{a_{j}}\left(b_{j}\right)\right)^{2}}{(2 \pi)^{-1}\left|J\left(a_{j}\right)\right|}\right)|d \xi| \\
& \geq 2^{-3} \epsilon_{0}\left(\frac{\min \left\{1,\left(1-s_{0}^{2}\right)^{1-p}\right\}}{\left(1-s^{2}\right)^{1-p}}\right),
\end{aligned}
$$

a contradiction. In other words, (12) must be true under (4) being valid.

Proof of Theorem 2. Suppose that $C_{\phi}: C \mathcal{A}_{p} \mapsto C \mathcal{A}_{q}$ is compact. Of course, this operator is bounded, and thus (1) holds. Choosing $b=\phi(a)$, we see that $f_{b}$ defined in Lemma 1 tends to 0 uniformly on compact subsets of $\mathbb{D}$ whenever $|b| \rightarrow 1$. Thus, $\lim _{|b| \rightarrow 1}\left\|C_{\phi} f_{b}\right\|_{C \mathcal{A}_{p}, *}=0$. As an immediate by-product of the C-part in the proof of Theorem 11, we have

$$
0=\lim _{|b| \rightarrow 1}\left\|C_{\phi} f_{b}\right\|_{C \mathcal{A}_{p}, *}^{2} \gtrsim \lim _{|b| \rightarrow 1} \frac{\left(1-|a|^{2}\right)^{1-q}|b|^{2}}{\left(1-|b|^{2}\right)^{1-p}}\left\|\sigma_{b} \circ \phi \circ \sigma_{a}\right\|_{2}^{2},
$$

whence deriving (4).

Next, we deal with the converse part of Theorem 2 according to $(p, q) \in$ $[0,2) \times[1,1]$ and $(p, q) \in(1,2) \times[0,2)$. In order to verify that $C_{\phi}: C \mathcal{A}_{p} \mapsto$ $C \mathcal{A}_{q}$ is a compact operator, it suffices to check that $\lim _{n \rightarrow \infty}\left\|C_{\phi} f_{n}\right\|_{C \mathcal{A}_{q}, *}=0$ holds for any sequence $\left(f_{n}\right)_{n=1}^{\infty}$ in $C \mathcal{A}_{p}$ with $\left\|f_{n}\right\|_{C \mathcal{A}_{p}, *} \leq 1$ and $f_{n} \rightarrow 0$ on compact subsets of $\mathbb{D}$ as $n \rightarrow \infty$. 
Situation 1 - assume that (1) holds and (4) is valid for $(p, q) \in[0,2) \times$ $[1,1]$. Upon writing

$$
\left\|C_{\phi} f_{n}\right\|_{C \mathcal{A}_{q}, *}^{2} \lesssim \sup _{|\phi(a)|>s} \mathrm{~T}(n, a, q)+\sup _{|\phi(a)| \leq s} \mathrm{~T}(n, a, q),
$$

where

$$
0<s<1 \& \mathrm{~T}(n, a, q)=\left(1-|a|^{2}\right)^{1-q}\left\|f_{n} \circ \phi \circ \sigma_{a}-f_{n} \circ \phi(a)\right\|_{2}^{2},
$$

we have to control $\sup _{|\phi(a)|>s} \mathrm{~T}(n, a, q)$ and $\sup _{|\phi(a)| \leq s} \mathrm{~T}(n, a, q)$ from above. To do so, set

$$
\left\{\begin{array}{l}
f_{n, a}=f_{n} \circ \phi \circ \sigma_{a}-f_{n}(\phi(a)) \\
g_{n, a}=f_{n} \circ \sigma_{\phi(a)}-f_{n}(\phi(a)) ; \\
\psi_{a}=\sigma_{\phi(a)} \circ \phi \circ \sigma_{a} ; \\
E(\phi, a, t)=\left\{\xi \in \mathbb{T}:\left|\sigma_{\phi(a)} \circ \phi \circ \sigma_{a}(\xi)\right|>t\right\} .
\end{array}\right.
$$

Using (9) we obtain

$$
\begin{aligned}
& \sup _{|\phi(a)|>s} \mathrm{~T}(n, a, q) \\
& \approx \sup _{|\phi(a)|>s}\left(1-|a|^{2}\right)^{1-q}\left\|f_{n, a}\right\|_{2}^{2} \\
& \lesssim \sup _{|\phi(a)|>s}\left(1-|a|^{2}\right)^{1-q}\left\|g_{n, a}\right\|_{2}^{2}\left\|\psi_{a}\right\|_{2}^{2} \\
& \lesssim \sup _{|\phi(a)|>s} \frac{\left(1-|a|^{2}\right)^{1-q}}{\left(1-|\phi(a)|^{2}\right)^{1-p}}\left\|\psi_{a}\right\|_{2}^{2}\left\|f_{n}\right\|_{C \mathcal{A}_{p}, *}^{2} \\
& \lesssim \sup _{|\phi(a)|>s} \frac{\left(1-|a|^{2}\right)^{1-q}}{\left(1-|\phi(a)|^{2}\right)^{1-p}}\left\|\psi_{a}\right\|_{2}^{2},
\end{aligned}
$$

whence getting by (4)

$$
\lim _{s \rightarrow 1} \sup _{|\phi(a)|>s} \mathrm{~T}(n, a, q)=0 \quad \forall \quad n=1,2,3, \ldots
$$

Meanwhile,

$$
\sup _{|\phi(a)| \leq s} \mathrm{~T}(n, a, q) \lesssim \sup _{|\phi(a)| \leq s} \mathrm{~T}_{1}(n, a, q)+\sup _{|\phi(a)| \leq s} \mathrm{~T}_{2}(n, a, q),
$$

where

$$
\left\{\begin{array}{l}
\mathrm{T}_{1}(n, a, q)=\left(1-|a|^{2}\right)^{1-q} \int_{\mathbb{T} \backslash E(\phi, a, t)}\left|f_{n, a}(\xi)\right|^{2}|d \xi| \\
\mathrm{T}_{2}(n, a, q)=\left(1-|a|^{2}\right)^{1-q} \int_{E(\phi, a, t)}\left|f_{n, a}(\xi)\right|^{2}|d \xi| .
\end{array}\right.
$$

Applying Schwarz's lemma to $g_{n, a}$ or using [6, (3.19)] we get

$$
\sup _{|z| \leq t}|z|^{-1}\left|g_{n, a}(z)\right| \leq 2 \sup _{|w| \leq t}\left|g_{n, a}(w)\right|
$$


thereby deriving

$$
\begin{aligned}
& \sup _{|\phi(a)| \leq s} \mathrm{~T}_{1}(n, a, q) \\
& \lesssim \sup _{|\phi(a)| \leq s}\left(1-|a|^{2}\right)^{1-q} \sup _{|w| \leq t}\left|g_{n, a}(w)\right|^{2} \int_{\mathbb{T}}\left|\psi_{a}(\xi)\right|^{2}|d \xi| \\
& \lesssim\left(1+(1-s)^{p-1}\right) \sup _{|w| \leq t}\left|g_{n, a}(w)\right|^{2} \sup _{|\phi(a)| \leq s} \frac{\left(1-|a|^{2}\right)^{1-q}}{\left(1-|\phi(a)|^{2}\right)^{1-p}}\left\|\psi_{a}\right\|_{2}^{2} \\
& \rightarrow 0 \text { as } n \rightarrow \infty,
\end{aligned}
$$

in which $|\phi(a)| \leq s$ and $|w| \leq t$ have been used. Also, a combination of (9), (11) and $q=1$ gives that if

$$
\left\{\begin{array}{l}
\lambda=(a \bar{b}-1) /(1-b \bar{a}) \\
\tau=\phi \circ \sigma_{a} \\
c=\sigma_{b}(a) \\
b \in \mathbb{D}
\end{array}\right.
$$

then

$$
\begin{aligned}
& \left\|f_{n, a}\right\|_{\mathcal{A}_{q}, *}^{2} \\
& =\sup _{b \in \mathbb{D}}\left(1-|b|^{2}\right)^{1-q}\left\|f_{n} \circ \tau \circ \sigma_{b}-f_{n} \circ \tau(b)\right\|_{2}^{2} \\
& \lesssim \sup _{b \in \mathbb{D}}\left(1-|b|^{2}\right)^{1-q}\left\|f_{n} \circ \sigma_{\tau(b)}-f_{n} \circ \tau(b)\right\|_{2}^{2}\left\|\sigma_{\tau(b)} \circ \tau \circ \sigma_{b}\right\|_{2}^{2} \\
& \lesssim\left\|f_{n}\right\|_{C \mathcal{A}_{p}, *}^{2} \sup _{b \in \mathbb{D}} \frac{\left(1-|b|^{2}\right)^{1-q}}{\left(1-|\tau(b)|^{2}\right)^{1-p}}\left\|\sigma_{\tau(b)} \circ \tau \circ \sigma_{b}\right\|_{2}^{2} \\
& \lesssim \sup _{c \in \mathbb{D}} \frac{\left(1-|\lambda c|^{2}\right)^{1-q}}{\left(1-|\phi(\lambda c)|^{2}\right)^{1-p}}\left\|\sigma_{\phi(\lambda c)} \circ \phi \circ\left(\lambda \sigma_{c}\right)\right\|_{2}^{2} \\
& \lesssim \sup _{c \in \mathbb{D}} \frac{\left(1-|c|^{2}\right)^{1-q}}{\left(1-|\phi(c)|^{2}\right)^{1-p}}\left\|\psi_{c}\right\|_{2}^{2} \\
& <\infty,
\end{aligned}
$$


and hence from the Cauchy-Schwarz inequality, Lemmas 3 , and $q=1$ it follows that

$$
\begin{aligned}
& \sup _{|\phi(a)| \leq s} \mathrm{~T}_{2}(n, a, q) \\
& \lesssim \sup _{|\phi(a)| \leq s}\left(1-|a|^{2}\right)^{1-q}\left(\int_{E(\phi, a, t)}\left|f_{n, a}(\xi)\right|^{4}|d \xi|\right)^{\frac{1}{2}}|E(\phi, a, t)|^{\frac{1}{2}} \\
& \lesssim \sup _{|\phi(a)| \leq s}\left(\frac{\left(1-|a|^{2}\right)^{1-q}}{\left(1-|\phi(a)|^{2}\right)^{p-1}} \int_{\mathbb{T}}\left|f_{n, a}(\xi)\right|^{4}|d \xi|\right)^{\frac{1}{2}}\left(\frac{\left(1-|a|^{2}\right)^{1-q}}{\left(1-|\phi(a)|^{2}\right)^{1-p}}|E(\phi, a, t)|\right)^{\frac{1}{2}} \\
& \lesssim\left(1+\left(1-s^{2}\right)^{1-p}\right)^{\frac{1}{2}} \sup _{|\phi(a)| \leq s}\left(\frac{\left\|f_{n, a}\right\|_{2}^{2}|| f_{n, a} \|_{\mathcal{C A}_{q}, *}^{2}}{\left(1-|a|^{2}\right)^{q-1}}\right)^{\frac{1}{2}}\left(\frac{|E(\phi, a, t)|}{\frac{\left(1-|\phi(a)|^{2}\right)^{1-p}}{\left(1-|a|^{2}\right)^{1-q}}}\right)^{\frac{1}{2}} \\
& \lesssim\left(1+\left(1-s^{2}\right)^{1-p}\right)^{\frac{1}{2}}\left\|f_{n, a}\right\|_{C \mathcal{A}_{q, *}}^{2} \sup _{|\phi(a)| \leq s}\left(\frac{\left(1-|a|^{2}\right)^{1-q}}{\left(1-|\phi(a)|^{2}\right)^{1-p}|E(\phi, a, t)|}\right)^{\frac{1}{2}} \\
& \lesssim\left(\frac { \operatorname { s u p } _ { c \in \mathbb { D } } \frac { ( 1 - | c | ^ { 2 } ) ^ { 1 - q } } { ( 1 - | \phi ( c ) | ^ { 2 } ) ^ { 1 - p } } \| \psi _ { c } \| _ { 2 } ^ { 2 } } { ( 1 + ( 1 - s ^ { 2 } ) ^ { 1 - p } ) ^ { - \frac { 1 } { 2 } } } \operatorname { s u p } _ { | \phi ( a ) | \leq s } \left(\frac{\left(1-|a|^{2}\right)^{1-q}}{\left.\left(1-|\phi(a)|^{2}\right)^{1-p}|E(\phi, a, t)|\right)^{\frac{1}{2}}}\right.\right. \\
& \rightarrow 0 \quad \text { as } t \rightarrow 1 .
\end{aligned}
$$

Consequently,

$$
\lim _{n \rightarrow \infty} \sup _{|\phi(a)| \leq s} \mathrm{~T}(n, a, q)=0 .
$$

Putting (17) and (18) together, we reach $\lim _{n \rightarrow \infty}\left\|C_{\phi} f_{n}\right\|_{C \mathcal{A}_{q, *}}=0$.

Situation 2 - assume that (1) holds and (4) is valid for $(p, q) \in(1,2) \times$ $[0,2)$. Rewriting

$$
\begin{aligned}
& \left\|C_{\phi} f_{n}\right\|_{\mathcal{C A}_{q}, *}^{2} \\
& \lesssim \sup _{a \in \mathbb{D}}\left(1-|a|^{2}\right)^{1-q} \int_{\mathbb{D}}\left|f_{n}^{\prime}(w)\right|^{2} N\left(\phi \circ \sigma_{a}, w\right) d m(w) \\
& \leq \sup _{a \in \mathbb{D}} \bigcup(n, a, q, r)+\sup _{a \in \mathbb{D}} \mathrm{V}(n, a, q, r),
\end{aligned}
$$

where $2^{-1} \leq r<1$ and

$$
\left\{\begin{array}{l}
\mathrm{U}(n, a, q, r)=\left(1-|a|^{2}\right)^{1-q} \int_{\left|\sigma_{\phi(a)}(w)\right| \leq r}\left|f_{n}^{\prime}(w)\right|^{2} N\left(\phi \circ \sigma_{a}, w\right) d m(w) ; \\
\mathrm{V}(n, a, q, r)=\left(1-|a|^{2}\right)^{1-q} \int_{\left|\sigma_{\phi(a)}(w)\right|>r}\left|f_{n}^{\prime}(w)\right|^{2} N\left(\phi \circ \sigma_{a}, w\right) d m(w),
\end{array}\right.
$$

we have to control $\sup _{a \in \mathbb{D}} \mathrm{U}(n, a, q, r)$ and $\sup _{a \in \mathbb{D}} \mathrm{V}(n, a, q, r)$ for an appropriate $r \in\left[2^{-1}, 1\right)$. In the sequel, let $b=\phi(a)$.

Sub-situation 1 - estimate for $\sup _{a \in \mathbb{D}} \mathrm{U}(n, a, q, r)$. For this, we consider two cases for any given $s \in(0,1)$. 
Case $1_{1}:|b| \leq s$. Under this case, $\left|\sigma_{b}(w)\right| \leq r$ ensures that $w$ belongs to a compact subset $K$ of $\mathbb{D}$, and therefore, it follows from $f_{n} \rightarrow 0$ on any compact subset of $\mathbb{D}$ and (11) that $\lim _{n \rightarrow \infty} \sup _{w \in K}\left|f_{n}^{\prime}(w)\right|=0$ and consequently,

$$
\begin{aligned}
& \lim _{n \rightarrow \infty} \sup _{|b| \leq s}\left(1-|a|^{2}\right)^{1-q} \int_{\left|\sigma_{b}(w)\right| \leq r}\left|f_{n}^{\prime}(w)\right|^{2} N\left(\phi \circ \sigma_{a}, w\right) d m(w) \\
& =\lim _{n \rightarrow \infty} \sup _{|b| \leq s} \frac{\left(1-|a|^{2}\right)^{1-q}}{\left(1-|b|^{2}\right)^{1-p}}\left(1-|b|^{2}\right)^{p-1} \int_{\left|\sigma_{b}(w)\right| \leq r}\left|f_{n}^{\prime}(w)\right|^{2} N\left(\phi \circ \sigma_{a}, w\right) d m(w) \\
& \lesssim\left(\lim _{n \rightarrow \infty} \sup _{w \in K}\left|f_{n}^{\prime}(w)\right|^{2}\right) \sup _{|b| \leq s} \frac{\left(1-|a|^{2}\right)^{1-q}}{\left(1-|b|^{2}\right)^{1-p}} \int_{\left|\sigma_{b}(w)\right| \leq r} N\left(\phi \circ \sigma_{a}, w\right) d m(w) \\
& \lesssim\left(\lim _{n \rightarrow \infty} \sup _{w \in K}\left|f_{n}^{\prime}(w)\right|^{2}\right) \sup _{|b| \leq s} \frac{\left(1-|a|^{2}\right)^{1-q}}{\left(1-|b|^{2}\right)^{1-p}} \int_{\mathbb{D}} N\left(\sigma_{b} \circ \phi \circ \sigma_{a}, z\right) d m(z) \\
& \lesssim\left(\lim _{n \rightarrow \infty} \sup _{w \in K}\left|f_{n}^{\prime}(w)\right|^{2}\right) \sup _{|b| \leq s} \frac{\left(1-|a|^{2}\right)^{1-q}}{\left(1-|b|^{2}\right)^{1-p}}\left\|\sigma_{b} \circ \phi \circ \sigma_{a}\right\|_{2}^{2} \\
& =0 .
\end{aligned}
$$

Case $1_{2}:|b|>s$. Using (8) we get

$$
\begin{aligned}
& \sup _{|b|>s}\left(1-|a|^{2}\right)^{1-q} \int_{\left|\sigma_{b}(w)\right| \leq r}\left|f_{n}^{\prime}(w)\right|^{2} N\left(\phi \circ \sigma_{a}, w\right) d m(w) \\
& \lesssim\left\|f_{n}\right\|_{C \mathcal{A}_{p}, *}^{2} \sup _{|b|>s}\left(1-|a|^{2}\right)^{1-q} \int_{\left|\sigma_{b}(w)\right| \leq r} N\left(\sigma_{b} \circ \phi \circ \sigma_{a}, \sigma_{b}(w)\right) \frac{d m(w)}{\left(1-|w|^{2}\right)^{3-p}} \\
& \lesssim \sup _{|b|>s}\left(1-|a|^{2}\right)^{1-q} \int_{|z| \leq r}\left(1-\left|\sigma_{b}(z)\right|^{2}\right)^{p-1} N\left(\sigma_{b} \circ \phi \circ \sigma_{a}, z\right) \frac{d m(z)}{\left(1-|z|^{2}\right)^{2}} \\
& \lesssim \sup _{|b|>s} \frac{\left(1-|a|^{2}\right)^{1-q}}{\left(1-|b|^{2}\right)^{1-p}} \int_{|z| \leq r} N\left(\sigma_{b} \circ \phi \circ \sigma_{a}, z\right) \frac{d m(z)}{\left(1-|z|^{2}\right)^{2}} \\
& \lesssim\left(1-r^{2}\right)^{-2} \sup _{|b|>s} \frac{\left(1-|a|^{2}\right)^{1-q}}{\left(1-|b|^{2}\right)^{1-p}}\left\|\sigma_{b} \circ \phi \circ \sigma_{a}\right\|_{2}^{2} \\
& \rightarrow 0 \quad \text { as } \quad s \rightarrow 1 .
\end{aligned}
$$

Putting the above two cases together, we see that for any $\epsilon \in(0,1)$ there are two real numbers: $r_{0} \in\left[2^{-1}, 1\right) ; s_{0} \in(0,1)$, and a natural number $n_{0}$ such that $n \geq n_{0}$

$$
\sup _{a \in \mathbb{D}} \mathrm{U}\left(n, a, q, r_{0}\right) \leq \sup _{|b| \leq s_{0}} \mathrm{U}\left(n, a, q, r_{0}\right)+\sup _{|b|>s_{0}} \mathrm{U}\left(n, a, q, r_{0}\right)<\epsilon .
$$

Sub-situation 2 - estimate for $\sup _{a \in \mathbb{D}} \mathrm{V}(n, a, q, r)$. Like Sub-situation 1, two treatments are required. 
Case $2_{1}:|b| \leq s$. For this case, we need the following by-product of [15, Lemma 2.1]: if $\psi$ is an analytic self-map of $\mathbb{D}$ with $\psi(0)=0$ then

$$
\mathrm{W}=\sup _{0<|w|<1}|w|^{2} N(\psi, w)<\infty \Longrightarrow \sup _{2^{-1} \leq|w|<1} \frac{N(\psi, w)}{\ln |w|^{-1}} \leq 4(\ln 2)^{-1} \mathbf{W}
$$

Note that (1) and (4) imply respectively

$$
\sup _{a \in \mathbb{D}} \frac{\left(1-|a|^{2}\right)^{1-q}}{\left(1-|b|^{2}\right)^{1-p}} \sup _{0<\mid w<1}|w|^{2} N\left(\sigma_{b} \circ \phi \circ \sigma_{a}, w\right)<\infty
$$

and

$$
\lim _{|b| \rightarrow 1} \frac{\left(1-|a|^{2}\right)^{1-q}}{\left(1-|b|^{2}\right)^{1-p}} \sup _{0<\mid w<1}|w|^{2} N\left(\sigma_{b} \circ \phi \circ \sigma_{a}, w\right)=0
$$

thanks to the following (10) $)$-based mean value estimate for $N\left(\sigma_{w} \circ \sigma_{b} \circ \phi \circ\right.$ $\sigma_{a}, 0$ ) where $0<|w|<1$ (cf. [6, (2.9)]):

$$
\begin{aligned}
& |w|^{2} N\left(\sigma_{b} \circ \phi \circ \sigma_{a}, w\right) \\
& =|w|^{2} N\left(\sigma_{w} \circ \sigma_{b} \circ \phi \circ \sigma_{a}, 0\right) \\
& \lesssim \int_{|z|<|w|} N\left(\sigma_{w} \circ \sigma_{b} \circ \phi \circ \sigma_{a}, z\right) d m(z) \\
& \lesssim \int_{\mathbb{D}} N\left(\sigma_{b} \circ \phi \circ \sigma_{a}, \sigma_{w}(z)\right) d m(z) \\
& \approx \int_{\mathbb{D}}\left|\sigma_{w}^{\prime}(z)\right|^{2} N\left(\sigma_{b} \circ \phi \circ \sigma_{a}, z\right) d m(z) \\
& \approx\left\|\sigma_{w} \circ \sigma_{b} \circ \phi \circ \sigma_{a}-\sigma_{w} \circ \sigma_{b} \circ \phi \circ \sigma_{a}(0)\right\|_{2}^{2} \\
& \lesssim\left\|\sigma_{b} \circ \phi \circ \sigma_{a}\right\|_{2}^{2} .
\end{aligned}
$$


Thus, a combination of (20)-(21)-(22) and Hölder's inequality gives

$$
\begin{aligned}
& \sup _{|b| \leq s}\left(1-|a|^{2}\right)^{1-q} \int_{\left|\sigma_{b}(w)\right|>r}\left|f_{n}^{\prime}(w)\right|^{2} N\left(\phi \circ \sigma_{a}, w\right) d m(w) \\
& \approx \sup _{|b| \leq s}\left(1-|a|^{2}\right)^{1-q} \int_{\left|\sigma_{b}(w)\right|>r}\left|f_{n}^{\prime}(w)\right|^{2} N\left(\sigma_{b} \circ \phi \circ \sigma_{a}, \sigma_{b}(w)\right) d m(w) \\
& \lesssim \sup _{|b| \leq s}\left(1-|b|^{2}\right)^{1-p} \int_{\left|\sigma_{b}(w)\right|>r}\left|f_{n}^{\prime}(w)\right|^{2} N\left(\sigma_{b}, w\right) d m(w) \\
& \lesssim\left(1+\left(1-s^{2}\right)^{1-p}\right) \int_{\left|\sigma_{b}(w)\right|>r}\left|f_{n}^{\prime}(w)\right|^{2} N\left(\sigma_{b}, w\right) d m(w) \\
& \lesssim\left(1+\left(1-s^{2}\right)^{1-p}\right) \int_{|z|>r}\left|\left(f_{n} \circ \sigma_{b}\right)^{\prime}(z)\right|^{2}\left(1-|z|^{2}\right) d m(z) \\
& \lesssim\left(1+\left(1-s^{2}\right)^{1-p}\right)\left(\frac{\int_{|z|>r}\left|\left(f_{n} \circ \sigma_{b}\right)^{\prime}(z)\right|^{4}\left(1-|z|^{2}\right)^{4-p} d m(z)}{\left(\int_{|z|>r}\left(1-|z|^{2}\right)^{p-2} d m(z)\right)^{-1}}\right)^{1 / 2}
\end{aligned}
$$

Since $\left\|f_{n}\right\|_{C \mathcal{A}_{p}, *} \leq 1$ and $|b| \leq s<1$ ensure $\left\|f_{n} \circ \sigma_{b}\right\|_{C \mathcal{A}_{p}, *} \lesssim 1$, one concludes that $\left|\left(f_{n} \circ \sigma_{b}\right)^{\prime}(z)\right|^{2}\left(1-|z|^{2}\right) d m(z)$ is $p$-Carleson measure with norm relying on $s$ and so that $d \mu_{n}(z)=\left|\left(f_{n} \circ \sigma_{b}\right)^{\prime}(z)\right|^{2}\left(1-|z|^{2}\right)^{4-p} d m(z)$ is 3-Carleson measure with norm relying on $s$. Now, it follows from [16, Theorem 1.2] that

$$
\begin{aligned}
& \int_{|z|>r}\left|\left(f_{n} \circ \sigma_{b}\right)^{\prime}(z)\right|^{4}\left(1-|z|^{2}\right)^{4-p} d m(z) \\
& =\int_{|z|>r}\left|\left(f_{n} \circ \sigma_{b}\right)^{\prime}(z)\right|^{2} d \mu_{n}(z) \\
& \lesssim\left\|\mu_{n}\right\|_{\mathcal{C M}_{3}} \int_{\mathbb{D}}\left|\left(f_{n} \circ \sigma_{b}\right)^{\prime}(z)\right|^{2}\left(1-|z|^{2}\right) d m(z) \\
& \lesssim\left\|f_{n}\right\|_{\mathcal{C A}_{p}, *}^{4} \\
& \lesssim 1 .
\end{aligned}
$$

Note that

$$
\lim _{r \rightarrow 1} \int_{|z|>r}\left(1-|z|^{2}\right)^{p-2} d m(z)=0
$$

So

$$
\limsup _{r \rightarrow 1}\left(1-|a|^{2}\right)^{1-q} \int_{\left|\sigma_{b}(w)\right|>r}\left|f_{n}^{\prime}(w)\right|^{2} N\left(\phi \circ \sigma_{a}, w\right) d m(w)=0
$$

holds for any $n=1,2,3, \ldots$. 
Case $2_{2}:|b|>s$. Since (22) implies that for any $\epsilon \in(0,1)$ there is an $s_{0} \in(0,1)$ such that

$$
|b|>s_{0} \Longrightarrow \frac{\left(1-|a|^{2}\right)^{1-q}}{\left(1-|b|^{2}\right)^{1-p}} \sup _{0<|w|<1}|w|^{2} N\left(\sigma_{b} \circ \phi \circ \sigma_{a}, w\right)<\epsilon .
$$

Thus, (20) is applied once again to deduce that

$$
\begin{aligned}
& N\left(\phi \circ \sigma_{a}, w\right) \\
& =N\left(\sigma_{b} \circ \phi \circ \sigma_{a}, \sigma_{b}(w)\right) \\
& \lesssim \frac{\epsilon\left(1-|b|^{2}\right)^{1-p}}{\left(1-|a|^{2}\right)^{1-q}} \ln \left|\sigma_{b}(w)\right|^{-1} \\
& \approx \frac{\epsilon\left(1-|b|^{2}\right)^{1-p}}{\left(1-|a|^{2}\right)^{1-q}} N\left(\sigma_{b}, w\right) \\
& \text { as }\left|\sigma_{b}(w)\right|>r>2^{-1} .
\end{aligned}
$$

Consequently,

$$
\begin{aligned}
& \sup _{|b|>s_{0}}\left(1-|a|^{2}\right)^{1-q} \int_{\left|\sigma_{b}(w)\right|>r}\left|f_{n}^{\prime}(w)\right|^{2} N\left(\phi \circ \sigma_{a}, w\right) d m(w) \\
& \lesssim \epsilon \sup _{|b|>s_{0}}\left(1-|b|^{2}\right)^{1-p} \int_{\left|\sigma_{b}(w)\right|>r}\left|f_{n}^{\prime}(w)\right|^{2} N\left(\sigma_{b}, w\right) d m(w) \\
& \lesssim \epsilon\left\|f_{n}\right\|_{C \mathcal{A}_{p}, *}^{2} \\
& \lesssim \epsilon .
\end{aligned}
$$

The previous discussions on Case $2_{1}$ and Case $2_{2}$ indicate

$$
\lim _{n \rightarrow \infty} \sup _{a \in \mathbb{D}} \mathrm{V}(n, a, q, r)=0 \text {. }
$$

Obviously, (19) and (23) give $\lim _{n \rightarrow \infty}\left\|C_{\phi} f_{n}\right\|_{C \mathcal{A}_{q, *}}=0$.

Proof of Corollary 2 This follows from (7), Theorem 2 and [20, Theorem $1.4(\mathrm{c})]$.

\section{REFERENCES}

[1] P. Bourdon, J. Cima and A. Matheson, Compact composition operators on the BMOA. Trans. Amer. Math. Soc. 351 (1999)2183-2196.

[2] C. Cascante, J. Fábrega and J. M. Ortega, The corona theorem in weighted Hardy and Morrey spaces. arXiv: 1006.5298v1 [math.CV]28June2010.

[3] P. M. Gauthier and J. Xiao, BiBloch-type maps: existence and beyond. In honor of Professor Erwin Kreyszig on the occasion of his 80th birthday. Complex Var. Theory Appl. 47(2002)667-678.

[4] M. Giaquinta, Multiple Integrals in the Calculus of Variations and Nonlinear Elliptic Systems. Ann. Math. Studies 105, Princeton University Press, Princeton, N.J., 1983. 
[5] H. Jarchow, Compactness properties of composition operators. International Workshop on Operator Theory (Cefalú, 1997). Rend. Circ. Mat. Palermo (2) Suppl. (1998)91-97.

[6] J. Laitila, Weighted composition operators on BMOA. Comput. Methods Funct. Th. 9(2009)27-46.

[7] J. Laitila, Isometric composition operators on BMOA. Math. Nachr. 283(2010)16461653.

[8] J. Laitila, P. J. Nieminen, E. Saksman and H.-O. Tylli, Compact and weakly compact composition operators on BMOA. arXiv:0912.3487v2 [math.FA] 22Jan2010.

[9] K. M. Madigan, Composition operators on analytic Lipschitz spaces. Proc. Amer. Math. Soc. 119(1993)465-473.

[10] K. Madigan and A. Matheson, Compact composition operators on the Bloch space. Trans. Amer. Math. Soc. 347(1995) 2679-2687.

[11] J. M. Orega and J. Fábrega, Pointwse multiplers and decomposition theorems in analytic Besov spaces. Math. Z. 235(2000)53-81.

[12] R. Roan, Composition operators on a space of Lipschitz functions. Rocky Mountain J. Math. 10(1980)371-379.

[13] J. H. Shapiro, Compact composition operators on spaces of boundary regular holomorphic functions. Proc. Amer. Math. Soc. 100(1987)49-57.

[14] J. H. Shapiro, Composition Operator and Classical Function Theory. SpringerVerlag, New York, 1993.

[15] W. Smith, Compactness of composition operators on BMOA. Proc. Amer. Math. Soc. 127(1999)2715-2725.

[16] D. A. Stegenga, Multipliers of the Dirichlet space. Illinois J. Math. 24(1980)114139.

[17] M. Tjani, Compact composition operators on Besov spaces. Trans. Amer. Math. Soc. 355(2003)4683-4698.

[18] H. Wulan, Compactness of composition operators on BMOA and VMOA. Sci. China Ser. A: Math. 50(2007)997-1004.

[19] H. Wulan, D. Zheng and K. Zhu, Compact composition operators on BMOA and the Bloch space. Proc. Amer. Math. Soc. 137(2009)3861-3868.

[20] J. Xiao, Composition operators associated with Bloch-type spaces. Complex Variables Theory Appl. 46(2001)109-121.

[21] J. Xiao, Holomorphic Q Classes. Lecture Notes in Math. 1767, Springer-Verlag, Berlin, 2001.

[22] J. Xiao, Geometric $Q_{p}$ Functions. Birkhauser-Verlag, 2006.

[23] K. Zhu, Operator Theory in Function Spaces. Math. Surveys and Monographs, Vol. 138, Amer. Math. Soc., 2007.

Department of Mathematics \& Statistics, Memorial University, NL A1C 5S7, Canada

E-mail address: jxiao@mun.ca; wenxupine@gmail.com 